\title{
The Weddell Sea region: an important precipitation channel to the interior of the Antarctic ice sheet as revealed by glaciochemical investigation of surface snow along the longest trans-Antarctic route
}

\author{
Qin Dahe, ${ }^{1}$ Paul A. Mayewhin,${ }^{2}$ Ren Jianen,,${ }^{1}$ Xiao Gunde,,${ }^{1}$ Sun Junying ${ }^{1}$ \\ ${ }^{1}$ Laboratory of Ice Core and Cold Regions Environment, Lanzhou Institute of \\ Glaciology and Geocryology, Chinese Academy of Sciences, Lanzhou, Gansu 730000, China \\ ${ }^{2}$ Climate Change Research Center, Institute for the Study of Earth, Oceans and Space, University of \\ New Hampshire, Durham, NH 03824, U.S.A
}

\begin{abstract}
Glaciochemical analysis of surface snow samples, collected along a profile crossing the Antarctic ice sheet from the Larsen Ice Shelf, Antarctic Peninsula, via the Antarctic Plateau through South Pole, Vostok and Komsomolskaya to Mirny station (at the east margin of East Antarctica), shows that the Weddell Sea region is an important channel for air masses to the high plateau of the Antarctic ice sheet $(>2000 \mathrm{~m}$ a.s.l.). This opinion is supported by the following. (1) The fluxes of sea-salt ions such as $\mathrm{Na}^{+}, \mathrm{Mg}^{2+}$ and $\mathrm{Cl}^{-}$display a decreasing trend from the west to the east of interior Antarctica. In general, as sea-salt aerosols are injected into the atmosphere over the Antarctic ice sheet from the Weddell Sea, large aerosols tend to decrease. For the inland plateau, few large particles of sea-salt aerosol reach the area, and the sea-salt concentration levels are low. (2) The high altitude of the East Antarctic plateau, as well as the polar cold high-pressure system, obstruct the intrusive air masses mainly from the South Indian Ocean sector. (3) For the coastal regions of the East Antarctic ice sheet, the elevation rises to $2000 \mathrm{~m}$ over a distance from several to several tens of $\mathrm{km}$. High concentrations of sea salt exist in snow in East Antarctica but are limited to a narrow coastal zone. (4) Fluxes of calcium and non-sea-salt sulfate in snow from the interior plateau do not display an eastward-decreasing trend. Since calcium is mainly derived from crustal sources, and $\mathrm{nssSO}_{4}{ }^{2-}$ is a secondary aerosol, this again confirms that the eastward-declining tendency of sea-salt ions indicates the transfer direction of precipitation vapor.
\end{abstract}

\section{INTRODUCTION}

Glaciochemical studies of the surface layer over the Antarctic ice sheet are still relatively rare. Previous studies have shown the basic principles governing the chemical composition of impurities in snow on the Antarctic ice sheet, and have provided an understanding of their origins and historic variations (Legrand and Delmas, 1985; Legrand, 1987; Kamiyama and others, 1989; Mayewski and Legrand, 1990; Mulvaney and Wolff, 1994). Since most of the data for the above studies were collected by different methods, procedures and investigators in different years, comparing the data is not always straightforward.

The 1990 International Trans-Antarctic Expedition (ITAE) provided a rare opportunity to investigate the surface characteristics of the Antarctic ice sheet over a wide region. Mulvaney and Wolff (1994) have compiled reliable data for $\mathrm{Na}^{+}, \mathrm{NO}_{3}{ }^{-}, \mathrm{Cl}^{-}$and $\mathrm{SO}_{4}{ }^{2-}$, mostly derived from several transects from the coast towards the interior. Up to now, such data are still very scarce for the vast interior region. The sampling sites along the ITAE route represent the longest transect ever conducted through the main geographic regions of Antarctica (Fig. 1). Along the ITAE route,

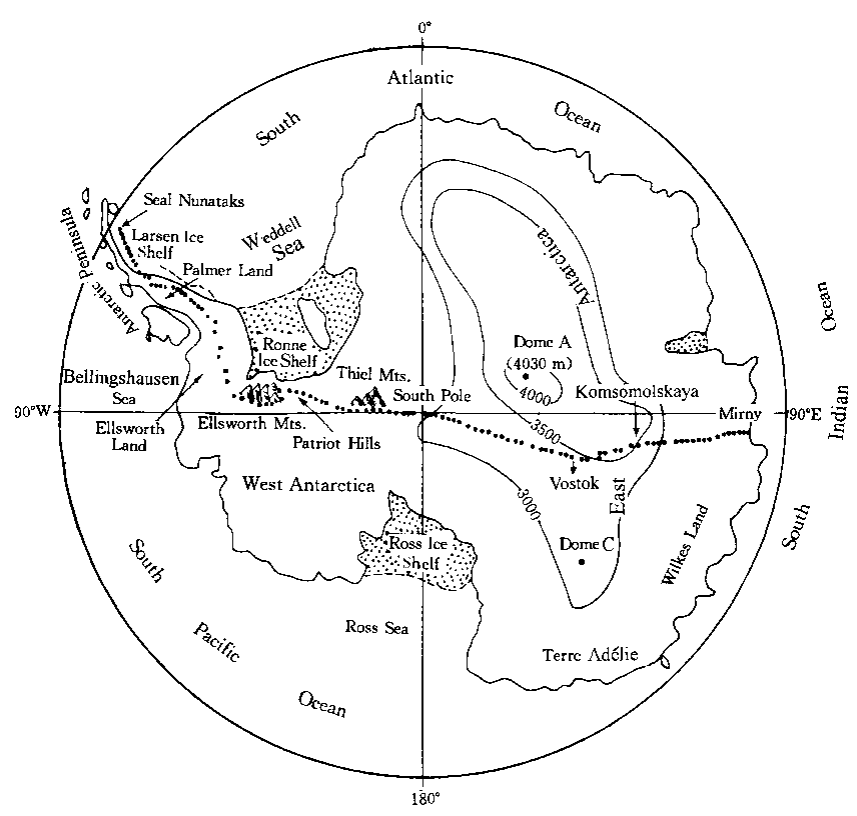

Fig. 1. Antarctic map showing the route of the 1990 ITAE and the sampling sites listed in Table 1. 
surface snow samples of $25 \mathrm{~cm}$ depth were collected at 97 sites. These samples were analyzed for major ions $\left(\mathrm{Na}^{+}\right.$, $\mathrm{Cl}^{-}, \mathrm{Mg}^{2+}, \mathrm{Ca}^{2+}, \mathrm{NO}_{3}{ }^{-}$and $\left.\mathrm{SO}_{4}{ }^{2-}\right)$ as well as methanesulfonic acid (MSA). An interpretation of the distribution of $\mathrm{NO}_{3}{ }^{-}$has been published (Qin and others, 1992). This paper presents the distribution of the major ions and MSA in the surface snow along the ITAE route, in order to provide a basic interpretation of snow chemistry along the study transect. This in turn casts new light on the problem of transportation of air masses onto the Antarctic ice sheet.

Understanding the mean annual temperature and accumulation rate along the ITAE route is essential for glaciochemical interpretation of the data collected. The mean annual temperature data at these sampling stations include (1) $-17.3^{\circ} \mathrm{C}$ measured in the firn at $9.5 \mathrm{~m}$ depth at station 10 on 31 August 1989; (2) temperatures measured previously at meteorological stations or in $10 \mathrm{~m}$ depth snow (at stations 23, 32, 52, 74, 82, 86 and 92 (Shimizu, 1964; Lorius and others, 1970; Schwerdtfeger, 1970; Barkov, 1975; Lipenkov, 1980; Jouzel and others, 1990) ); (3) estimated temperatures obtained from the isothermal diagram of average annual surface temperature in the interior of the East Antarctic plateau (Crary, 1963; Cameron and others, 1968); and (4) the lapse rate and the latitude effect. The elevation along the route was obtained from a large-scale contour map of Antarctica (Drewry, 1983), corrected to position records made on the traverse by G. Samers and J.-L. Etienne (personal communication, 1992).

The Larsen Ice Shelf, located on the east side of the Antarctic Peninsula and terminating as ice cliffs in the Weddell Sea, is in a continental climate region. This is because the Antarctic Peninsula is a natural meteorological barrier that demarcates a distinct climatic division between a maritime climate in the west and a continental climate in the east. Sampling sites 1-12 were set on the Larsen Ice Shelf. At these sites, elevations are below $100 \mathrm{~m}$, annual mean temperatures are $-12^{\circ}$ to $-14^{\circ} \mathrm{C}$ and the average accumulation rates are in the range $500-600 \mathrm{~kg} \mathrm{~m}^{-2} \mathrm{a}^{-1}$. Sites $13-19$ are distributed over Palmer Land in the Antarctic Peninsula. Within this section of the traverse route, topography is complicated, with an elevation range of several hundred meters to $2000 \mathrm{~m}$ and the distance to the coast changing irregularly. The estimated accumulation rates are $50-400 \mathrm{~kg} \mathrm{~m}^{-2} \mathrm{a}^{-1}$ and the annual mean temperatures range from $-16^{\circ}$ to $-22^{\circ} \mathrm{C}$.

From site 30 to the end of the traverse is the main section of the expedition route. Except for the Ellsworth Mountains, where elevation changes irregularly, the elevation increases progressively with increasing distance along the route to the highest point (site 86, $3560 \mathrm{~m}$ a.s.l.) and then decreases. About half of this section of the route is $>3000 \mathrm{~m}$ a.s.l., belonging to the interior of Antarctic Plateau. Here the climate is quite stable since it is under the control of the polar cold high-pressure system. The annual mean temperature is lowest $\left(-55^{\circ} \mathrm{C}\right)$ at Vostok, from where, toward both east and west, it increases $\left(-49^{\circ} \mathrm{C}\right.$ at South Pole and $-27^{\circ} \mathrm{C}$ at Patriot Hills). The accumulation is $<100 \mathrm{~kg} \mathrm{~m}^{-2} \mathrm{a}^{-1}$ at most sites within this section of the route, except at the Ellsworth Mountains. The accumulation and climatic data come from several different sources and are described in detail by Qin (1992). Variations in mean annual temperature, accumulation rate and altitude along the route are shown in Figure 2. The accumulation rate was largely derived from the compilation of Giovinetto and Bull (1987), but additional data were used from shallow ice cores and shallow snow pits which covered several tens of years and several years of snow accumulation, respectively (Shimi-
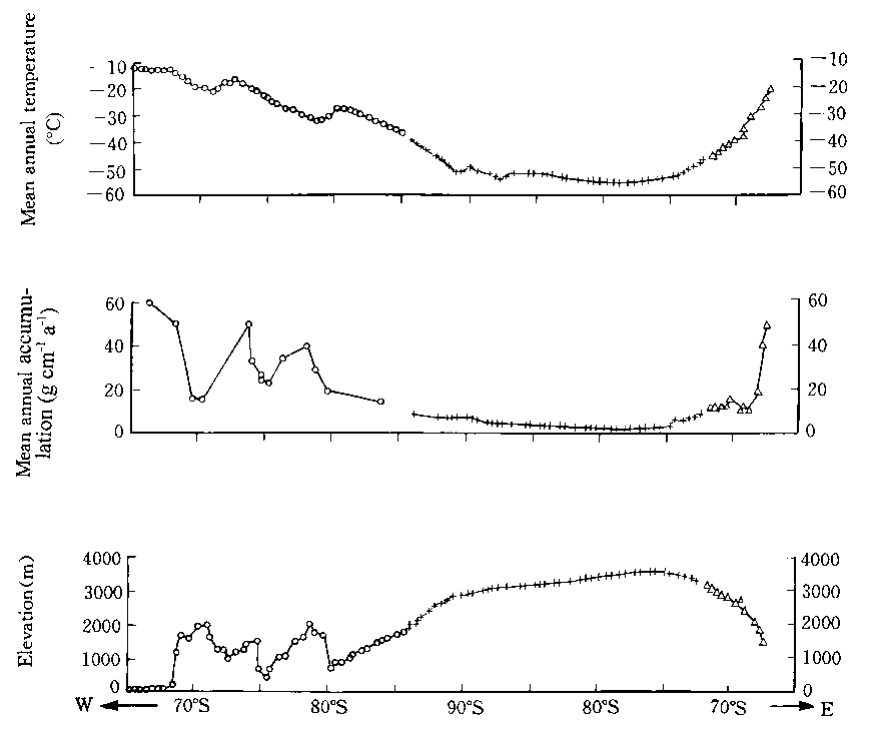

Fig. 2. Variations of mean annual temperature, snow-accumulation rate and altitude change along the ITAE route. The abscissa is the latitudes of the sampling sites.

zu, 1964; Taylor, 1965; Hamilton and O'Kelley, 1971; Kotlyakov and others, 1978; Lipenkov, 1980; Peel and Clausen, 1982; Jouzel and others, 1983).

Elements derived from the marine environment, $\mathrm{Na}^{+}$, $\mathrm{Cl}^{-}$and $\mathrm{Mg}^{2+}$, are used in this study as a proxy for vapor transport. Non-sea-salt derived elements such as $\mathrm{Ca}^{2+}$ and non-sea-salt sulfate $\left(\mathrm{nssSO}_{4}{ }^{2-}\right)$ are used to compare with the sea-salt distribution since they are tracers of continental source area and marine biogenic sources, respectively.

\section{SAMPLING AND ANALYTIGAL PROGEDURES}

From 27 July 1989 to 3 March 1990, the ITAE succeeded in crossing the Antarctic by dog-sled. The expedition route began at Seal Nunataks $\left(65^{\circ} 05^{\prime} \mathrm{S}, 59^{\circ} 35^{\prime} \mathrm{W}\right)$, then traversed from the northern end of the Antarctic Peninsula via the Larsen Ice Shelf, Palmer Land, the Ellsworth Mountains $\left(76-81^{\circ} \mathrm{S}, 87^{\circ} \mathrm{W}\right)$, South Pole, the Area of Inaccessibility and Vostok, and ended at Mirny, a station at the margin of East Antarctica. The route covered a total distance of $5896 \mathrm{~km}$ and the journey took 220 days. Ninety-seven $25 \mathrm{~cm}$ deep samples of surface snow were collected in polyethylene bottles along the route at roughly equidistant points. Special steps were taken to keep the samples as clean as possible during sampling. The bottles were new and were washed three times using Milli-Q water to clean each bottle to maintain blank values in the ppb range for ionic measurement of the snow samples. Washed bottles were packed in clean bags in a clean room and were opened only during sampling for a short time. Contamination was avoided by wearing gloves, cleaning the surface of the profile of the snow pits and facing in the windward direction when collecting samples.

All samples were kept frozen until processed. Concentrations of major anions and cations in the samples were measured by Dionex 4000 ion chromatography at the Laboratoire de Glaciologie et Géophysique de l'Environnement, Grenoble, France. The detection limits and precision, respectively, were $1 \times 10^{-6} \mathrm{~g} \mathrm{~g}^{-1}$ and $4 \%$ for $\mathrm{Cl}^{-}, 0.5 \times 10^{-6} \mathrm{~g} \mathrm{~g}^{-1}$ and $5 \%$ for $\mathrm{Na}^{+}$, $0.1 \times 10^{-6} \mathrm{~g} \mathrm{~g}^{-1}$ and $10 \%$ for $\mathrm{Mg}^{2+}, 0.5 \times 10^{-6} \mathrm{~g} \mathrm{~g}^{-1}$ and $50 \%$ for $\mathrm{Ca}^{2+}$, and $1 \times 10^{-6} \mathrm{~g} \mathrm{~g}^{-1}$ and $5 \%$ for $\mathrm{SO}_{4}{ }^{2-}$. 


\section{RESULTS AND DISCUSSION}

Analytical results for $\mathrm{Na}^{+}, \mathrm{Cl}^{-}, \mathrm{Mg}^{2+}, \mathrm{SO}_{4}{ }^{2-}$ and $\mathrm{Ca}^{2+}$ in samples are presented in Table 1 along with the sampling date and location. The distance from station 1 (Seal Nunataks), elevation and mean annual accumulation rate at 65 stations have been listed previously (Qin and others, 1992). Among the major ions, $\mathrm{Na}^{+}$is usually considered to be totally derived from sea-salt aerosol. Thus it is often selected for calculating sea-salt (ss) and non-sea-salt (nss) fractions of other ions. The calculation is important because it could provide information on sources, transportation and/or development of aerosol recorded in snow. Unfortunately, we find negative values of $n s s \mathrm{Mg}^{2+}$ when the calculation is based on $\mathrm{Na}^{+}$. Since we exclude the possibility that the samples were contaminated, the explanation is believed to be (1) $\mathrm{Na}^{+}$was concentrated during the process of transportation from source region to deposition site, and /or (2) extra

Table 1. The results of chemical analyses of some ions in $25 \mathrm{~cm}$ deep snow samples on the ITAE route. Unit of ion concentration is $10^{-6} \mathrm{~g} \mathrm{~g}^{-1}$. The sampling date, positions and represented seasons of the snow are also listed

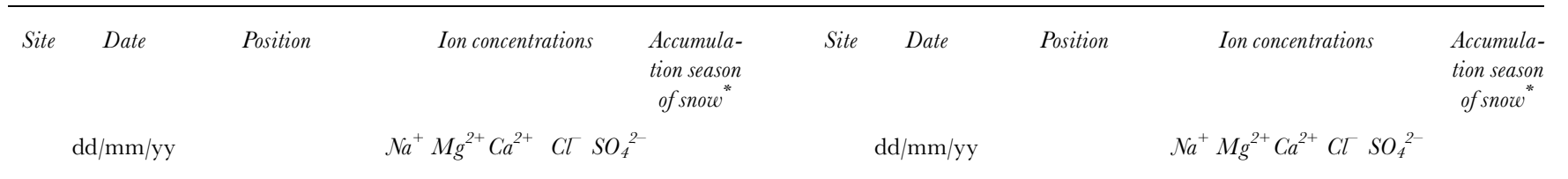

\begin{tabular}{|c|c|c|c|c|c|c|c|}
\hline 1 & $27 / 07 / 89$ & $65^{\circ} 05^{\prime} \mathrm{S} 59^{\circ} 35^{\prime} \mathrm{W}$ & 271 & 32.7 & 14.5 & 441 & 54 \\
\hline 2 & $28 / 07 / 89$ & $65^{\circ} 10^{\prime} \mathrm{S} 59^{\circ} 59^{\prime} \mathrm{W}$ & - & - & - & - & - \\
\hline 3 & $29 / 07 / 89$ & $65^{\circ} 17^{\prime} \mathrm{S} 60^{\circ} 19^{\prime} \mathrm{W}$ & - & - & - & - & - \\
\hline 4 & $30 / 07 / 89$ & $65^{\circ} 27^{\prime} \mathrm{S} 60^{\circ} 45^{\prime} \mathrm{W}$ & - & - & - & - & - \\
\hline 5 & 01/08/89 & $65^{\circ} 35^{\prime} \mathrm{S} \quad 61^{\circ} 10^{\prime} \mathrm{W}$ & 457 & 55.1 & 32.5 & 799 & 132 \\
\hline 6 & 02/08/89 & $65^{\circ} 42^{\prime} \mathrm{S} \quad 61^{\circ} 32^{\prime} \mathrm{W}$ & - & - & - & - & - \\
\hline 7 & 03/08/89 & $65^{\circ} 50^{\prime} \mathrm{S} \quad 61^{\circ} 57^{\prime} \mathrm{W}$ & 324 & 39.0 & 24.5 & 578 & 100 \\
\hline 8 & 10/08/89 & $66^{\circ} 20^{\prime} \mathrm{S} 62^{\circ} 36^{\prime} \mathrm{W}$ & 1054 & 127.0 & 48.5 & 1872 & 210 \\
\hline 9 & $13 / 08 / 89$ & $66^{\circ} 54^{\prime} \mathrm{S} 63^{\circ} 32^{\prime} \mathrm{W}$ & 499 & 60.1 & 23.0 & 784 & 112 \\
\hline 10 & $15 / 08 / 89$ & $67^{\circ} 20^{\prime} \mathrm{S} 64^{\circ} 07^{\prime} \mathrm{W}$ & 43 & 5.2 & 2.5 & 61 & 17 \\
\hline 11 & $18 / 08 / 89$ & $67^{\circ} 47^{\prime} \mathrm{S} 64^{\circ} 32^{\prime} \mathrm{W}$ & 21 & 2.5 & 2.0 & 32 & 12 \\
\hline 12 & $23 / 08 / 89$ & $68^{\circ} 25^{\prime} \mathrm{S} 65^{\circ} 15^{\prime} \mathrm{W}$ & 76 & 9.2 & 4.5 & 107 & 29 \\
\hline 13 & $27 / 08 / 89$ & $68^{\circ} 43^{\prime} \mathrm{S} 65^{\circ} 26^{\prime} \mathrm{W}$ & 60 & 7.2 & 4.0 & 115 & 31 \\
\hline 14 & $28 / 08 / 89$ & $68^{\circ} 48^{\prime} \mathrm{S} 65^{\circ} 22^{\prime} \mathrm{W}$ & - & - & - & - & - \\
\hline 15 & $29 / 08 / 89$ & $68^{\circ} 57^{\prime} \mathrm{S} 65^{\circ} 26^{\prime} \mathrm{W}$ & - & - & - & - & - \\
\hline 16 & $30 / 08 / 89$ & $69^{\circ} 04^{\prime} \mathrm{S} 65^{\circ} 20^{\prime} \mathrm{W}$ & - & - & - & - & - \\
\hline 17 & 31/08/89 & $69^{\circ} 10^{\prime} \mathrm{S} 65^{\circ} 18^{\prime} \mathrm{W}$ & 21 & 2.5 & 1.5 & 42 & 26 \\
\hline 18 & 05/09/89 & $69^{\circ} 41^{\prime} \mathrm{S} \quad 65^{\circ} 13^{\prime} \mathrm{W}$ & 12 & 1.5 & 1.0 & 23 & 29 \\
\hline 19 & 09/09/89 & $70^{\circ} 25^{\prime} \mathrm{S} 64^{\circ} 44^{\prime} \mathrm{W}$ & 25 & 3.0 & 2.0 & 73 & 47 \\
\hline 20 & $14 / 09 / 89$ & $70^{\circ} 58^{\prime} \mathrm{S} \quad 64^{\circ} 41^{\prime} \mathrm{W}$ & 7 & 0.8 & 1.0 & 17 & 20 \\
\hline 21 & $15 / 09 / 89$ & $71^{\circ} 17^{\prime} \mathrm{S} 65^{\circ} 43^{\prime} \mathrm{W}$ & 85 & 10.2 & 3.5 & 137 & 29 \\
\hline 22 & $17 / 09 / 89$ & $71^{\circ} 56^{\prime} \mathrm{S} 65^{\circ} 18^{\prime} \mathrm{W}$ & 31 & 3.7 & 3.5 & 64 & 22 \\
\hline 23 & $18 / 09 / 89$ & $72^{\circ} 14^{\prime} \mathrm{S} 65^{\circ} 29^{\prime} \mathrm{W}$ & 47 & 5.7 & 3.0 & 86 & 18 \\
\hline 24 & 19/09/89 & $72^{\circ} 33^{\prime} \mathrm{S} 65^{\circ} 55^{\prime} \mathrm{W}$ & 17 & 2.1 & 1.5 & 34 & 11 \\
\hline 25 & $23 / 09 / 89$ & $73^{\circ} 14^{\prime} \mathrm{S} 66^{\circ} 48^{\prime} \mathrm{W}$ & 54 & 6.5 & 2.5 & 98 & 18 \\
\hline 26 & $30 / 09 / 89$ & $73^{\circ} 56^{\prime} \mathrm{S} 67^{\circ} 30^{\prime} \mathrm{W}$ & 88 & 10.6 & 4.0 & 164 & 32 \\
\hline 27 & 06/10/89 & $74^{\circ} 05^{\prime} \mathrm{S} 69^{\circ} 57^{\prime} \mathrm{W}$ & 26 & 3.1 & 2.5 & 50 & 10 \\
\hline 28 & 09/10/89 & $74^{\circ} 46^{\prime} \mathrm{S} 72^{\circ} 46^{\prime} \mathrm{W}$ & 87 & 10.5 & 5.0 & 164 & 29 \\
\hline 29 & $11 / 10 / 89$ & $74^{\circ} 51^{\prime} \mathrm{S} 75^{\circ} 45^{\prime} \mathrm{W}$ & 51 & 6.1 & 2.5 & 89 & 30 \\
\hline 30 & $15 / 10 / 89$ & $75^{\circ} 26^{\prime} \mathrm{S} 78^{\circ} 29^{\prime} \mathrm{W}$ & 60 & 7.2 & 3.0 & 10 & 29 \\
\hline 31 & $18 / 10 / 89$ & $75^{\circ} 47^{\prime} \mathrm{S} 81^{\circ} 40^{\prime} \mathrm{W}$ & 66 & 8.0 & 4.0 & 111 & 28 \\
\hline 32 & $24 / 10 / 89$ & $76^{\circ} 25^{\prime} \mathrm{S} 85^{\circ} 10^{\prime} \mathrm{W}$ & 44 & 5.3 & 4.0 & 80 & 29 \\
\hline 33 & 26/10/89 & $76^{\circ} 56^{\prime} \mathrm{S} 86^{\circ} 15^{\prime} \mathrm{W}$ & 31 & 3.7 & 2.5 & 68 & 18 \\
\hline 34 & $28 / 10 / 89$ & $77^{\circ} 39^{\prime} \mathrm{S} 87^{\circ} 07^{\prime} \mathrm{W}$ & 38 & 4.6 & 4.0 & 78 & 18 \\
\hline 35 & $30 / 10 / 89$ & $78^{\circ} 12^{\prime} \mathrm{S} 87^{\circ} 39^{\prime} \mathrm{W}$ & 33 & 4.0 & 3.5 & 77 & 23 \\
\hline 36 & $01 / 11 / 89$ & $78^{\circ} 49^{\prime} \mathrm{S} 87^{\circ} 14^{\prime} \mathrm{W}$ & 165 & 20.0 & 7.0 & 264 & 30 \\
\hline 37 & $02 / 11 / 89$ & $79^{\circ} 10^{\prime} \mathrm{S} 86^{\circ} 51^{\prime} \mathrm{W}$ & 66 & 8.0 & 6.5 & 139 & 29 \\
\hline 38 & 04/11/89 & $79^{\circ} 45^{\prime} \mathrm{S} 85^{\circ} 18^{\prime} \mathrm{W}$ & 32 & 3.8 & 3.0 & 73 & 31 \\
\hline 39 & 09/11/89 & $80^{\circ} 18^{\prime} \mathrm{S} \quad 81^{\circ} 21^{\prime} \mathrm{W}$ & 109 & 13.1 & 75.5 & 97 & 94 \\
\hline 40 & $11 / 11 / 89$ & $80^{\circ} 45^{\prime} \mathrm{S} 81^{\circ} 15^{\prime} \mathrm{W}$ & 204 & 24.6 & 7.0 & 342 & 38 \\
\hline 41 & $14 / 11 / 89$ & $81^{\circ} 12^{\prime} \mathrm{S} 82^{\circ} 00^{\prime} \mathrm{W}$ & 304 & 36.6 & 8.5 & 444 & 42 \\
\hline 42 & $16 / 11 / 89$ & $81^{\circ} 48^{\prime} \mathrm{S} 82^{\circ} 50^{\prime} \mathrm{W}$ & 271 & 32.7 & 9.5 & 409 & 62 \\
\hline 43 & 17/11/89 & $81^{\circ} 55^{\prime} \mathrm{S} 83^{\circ} 20^{\prime} \mathrm{W}$ & 65 & 7.8 & 4.0 & 98 & 29 \\
\hline 44 & $19 / 11 / 89$ & $82^{\circ} 41^{\prime} \mathrm{S} 84^{\circ} 00^{\prime} \mathrm{W}$ & 156 & 18.8 & 6.5 & 235 & 41 \\
\hline 45 & 20/11/89 & $83^{\circ} 05^{\prime} \mathrm{S} 84^{\circ} 45^{\prime} \mathrm{W}$ & 70 & 8.4 & 7.5 & 170 & 35 \\
\hline 46 & $22 / 11 / 89$ & $83^{\circ} 47^{\prime} \mathrm{S} 87^{\circ} 25^{\prime} \mathrm{W}$ & 44 & 5.3 & 3.0 & 84 & 22 \\
\hline 47 & 23/11/89 & $84^{\circ} 12^{\prime} \mathrm{S} 88^{\circ} 05^{\prime} \mathrm{W}$ & 27 & 3.3 & 2.5 & 68 & 19 \\
\hline 48 & 24/11/89 & $84^{\circ} 35^{\prime} \mathrm{S} 88^{\circ} 55^{\prime} \mathrm{W}$ & 33 & 4.0 & 4.0 & 82 & 38 \\
\hline 49 & 26/11/89 & $85^{\circ} 11^{\prime} \mathrm{S} 88^{\circ} 58^{\prime} \mathrm{W}$ & 66 & 7.9 & 3.0 & 130 & 26 \\
\hline 50 & 29/11/89 & $85^{\circ} 53^{\prime} \mathrm{S} 88^{\circ} 10^{\prime} \mathrm{W}$ & 15 & 1.8 & 2.5 & 48 & 25 \\
\hline 51 & 30/11/89 & $86^{\circ} 12^{\prime} \mathrm{S} 88^{\circ} 25^{\prime} \mathrm{W}$ & 14 & 1.7 & 2.0 & 44 & 24 \\
\hline 52 & $02 / 12 / 89$ & $86^{\circ} 34^{\prime} \mathrm{S} 88^{\circ} 57^{\prime} \mathrm{W}$ & 17 & 2.0 & 3.5 & 66 & 29 \\
\hline 53 & 03/12/89 & $86^{\circ} 54^{\prime} \mathrm{S} 90^{\circ} 19^{\prime} \mathrm{W}$ & 17 & 2.0 & 1.5 & 49 & 72 \\
\hline
\end{tabular}

W

$-$

W

$-$

W

W

W

W

W

W

$-$

W

W

W

W

W

W

W

W

W

W

W

W

W

W

W

W

W

W

W

W

W

W

W

W

W

W

W

W

W

W

W

M

M

M

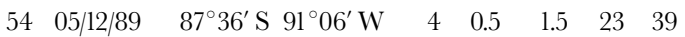

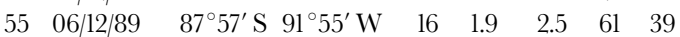

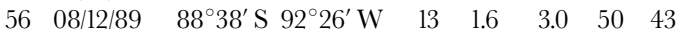

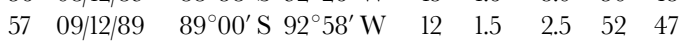

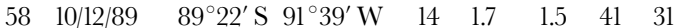

$\begin{array}{llllllll}59 & 13 / 12 / 89 & 90^{\circ} 00^{\prime} \mathrm{S} & 8 & 1.0 & 1.5 & 30 & 39\end{array}$

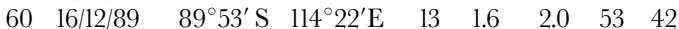

$\begin{array}{lllllllll}61 & 17 / 12 / 89 & 89^{\circ} 32^{\prime} \mathrm{S} & 108^{\circ} 18^{\prime} \mathrm{E} & 17 & 2.1 & 2.0 & 59 & 45\end{array}$

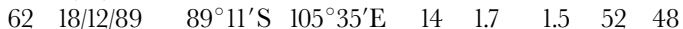

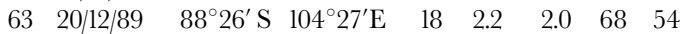

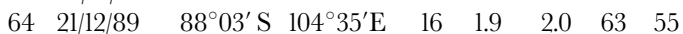

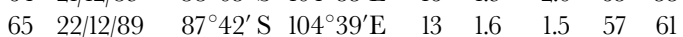

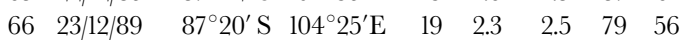

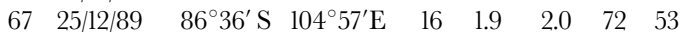

$\begin{array}{lllllllll}68 & 29 / 12 / 89 & 85^{\circ} 33^{\prime} \mathrm{S} & 105^{\circ} 40^{\prime} \mathrm{E} & 16 & 1.9 & 2.0 & 61 & 83\end{array}$

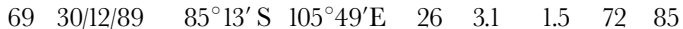

$\begin{array}{lllllllll}70 & 01 / 01 / 90 & 84^{\circ} 28^{\prime} \mathrm{S} & 106^{\circ} 17^{\prime} \mathrm{E} & 20 & 2.4 & 3.0 & 72 & 68\end{array}$

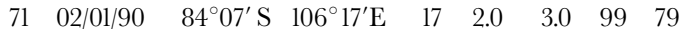

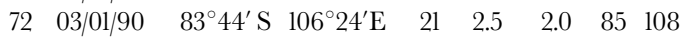

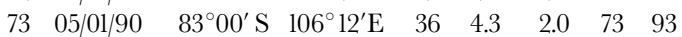

$\begin{array}{lllllllll}74 & 06 / 01 / 90 & 82^{\circ} 40^{\prime} \mathrm{S} & 106^{\circ} 19^{\prime} \mathrm{E} & 17 & 2.0 & 3.0 & 116 & 77\end{array}$

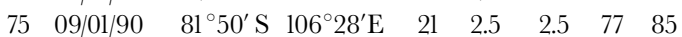

$\begin{array}{lllllllll}76 & 11 / 01 / 90 & 81^{\circ} 05^{\prime} \mathrm{S} & 106^{\circ} 26^{\prime} \mathrm{E} & - & - & - & - & 119\end{array}$

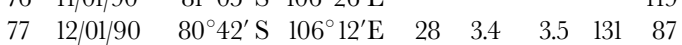

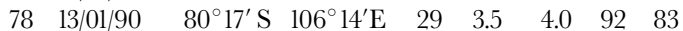

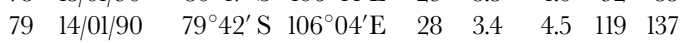

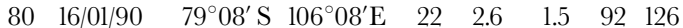

$\begin{array}{lllllllll}81 & 17 / 01 / 90 & 78^{\circ} 46^{\prime} \mathrm{S} & 106^{\circ} 41^{\prime} \mathrm{E} & 24 & 2.9 & 2.5 & 116 & 110\end{array}$

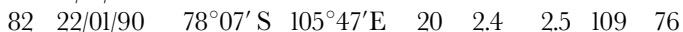

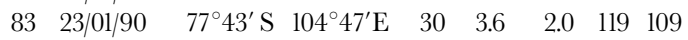

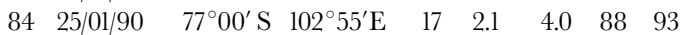

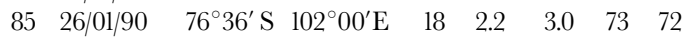

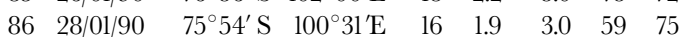

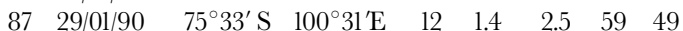

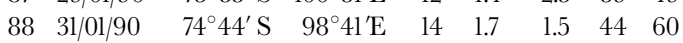

$\begin{array}{lllllllll}89 & 01 / 02 / 90 & 74^{\circ} 21^{\prime} \mathrm{S} & 98^{\circ} 00^{\prime} \mathrm{E} & 19 & 2.3 & 4.0 & 60 & 53\end{array}$

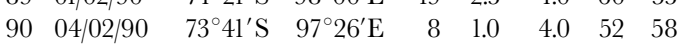

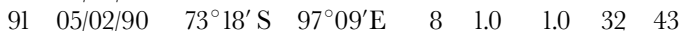

$\begin{array}{lllllllll}92 & 06 / 02 / 90 & 72^{\circ} 51^{\prime} \mathrm{S} & 96^{\circ} 59^{\prime} \mathrm{E} & 4 & 0.5 & 3.0 & 37 & 25\end{array}$

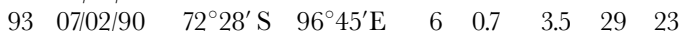

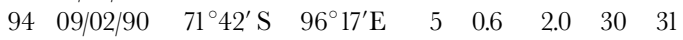

$\begin{array}{lllllllll} & 10 / 02 / 90 & 71^{\circ} 20^{\prime} \mathrm{S} & 96^{\circ} 01^{\prime} \mathrm{E} & 12 & 1.4 & 4.5 & 46 & 41\end{array}$

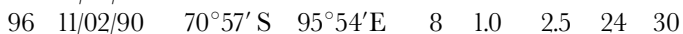

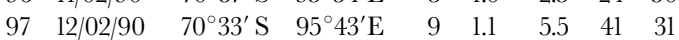

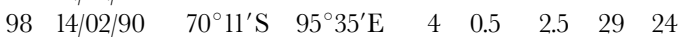

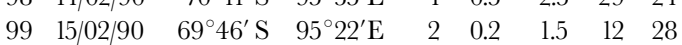

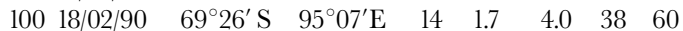

$10121 / 02 / 90 \quad 68^{\circ} 51^{\prime} \mathrm{S} \quad 94^{\circ} 37^{\prime} \mathrm{E} \quad 4 \quad 0.5 \quad 2.0 \quad 28 \quad 35$

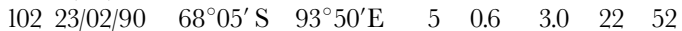

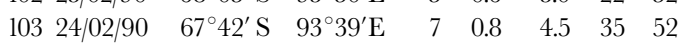

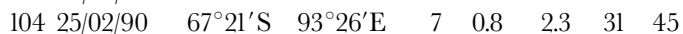

$105 \quad 03 / 03 / 90 \quad 66^{\circ} 33^{\prime} \mathrm{S} \quad 93^{\circ} 00^{\prime} \mathrm{E}$
M

$\mathrm{M}$

$\mathrm{M}$

$\mathrm{M}$

M

M

M

M

M

M

M

M

M

M

$\mathrm{M}$

M

$\mathrm{M}$

M

M

M

M

M

M

M

M

M

M

M

S

S

S

S

S

$\mathrm{S}$

$\mathrm{S}$

S

* W, winter snow; S, summer snow; M, multi-year snow. 
$\mathrm{Na}^{+}$, such as crustal-origin $\mathrm{Na}^{+}$, exists in addition to sea-salt aerosol. $\mathrm{Mg}^{2+}$ was therefore chosen for the calculation. The revised $\mathrm{Na}^{+}$concentrations are listed in Table 1 .

The number of years represented by the $25 \mathrm{~cm}$ snow samples depends on the accumulation rate at the sampling site. Generally, it is difficult to identify annual layers in such a shallow depth, so we estimate the accumulation rate as follows.

The net accumulation rate in Antarctica usually decreases with increasing latitude from the coast to the interior. But this relationship is less reliable than that of the mean annual temperature with latitude, particularly in near-coastal regions. On the other hand, accumulation rate may exhibit high annual variability. All of these circumstances make it difficult to estimate the age of the surface snow in Antarctica. However, Giovinetto and Bull (1987) summarized recent surface mass-balance measurements over the Antarctic ice sheet. By using previous records of accumulation rate at some sites on the route (e.g. sites 25, 37, 39, 62, 64, 66, 68, as well as sites 90-104 (Shimizu, 1964; Taylor, 1965; Hamilton and others, 1971; Lipenkov, 1980, Peel and Clausen, 1982; Jouzel and others, 1983, 1990), and by interpolating values between the sites at which accumulation rate is known, the age of the surface $1 \mathrm{~m}$ snow along the traverse route was plotted (Qin and others, 1994).

The pattern of the mean annual accumulation rate along the ITAE route is one of high accumulation at the beginning and end of the route, and lower accumulation in the middle section (Qin and others, 1992). The highest value is $600 \mathrm{~kg} \mathrm{~m}^{-2} \mathrm{a}^{-1}$, and the lowest is $23 \mathrm{~kg} \mathrm{~m}^{-2} \mathrm{a}^{-1}$. Using a mean snow density of $400 \mathrm{~kg} \mathrm{~m}^{-3}$ in the regions where the accumulation rate is $<100 \mathrm{~kg} \mathrm{~m}^{-2} \mathrm{a}^{-1}$, a $25 \mathrm{~cm}$ depth snow sample may represent 1-4 annual layers (Fig. 3), and we call this multi-year snow ( $\mathrm{M}$ in Table 1, sites $51-93$ ). On the east side of section $\mathrm{M}$ (i.e. from Seal Nunataks to site 50), the surface $25 \mathrm{~cm}$ represents only winter snow ( $\mathrm{W}$ in Table 1 ) since the accumulation rate is $>100 \mathrm{~kg} \mathrm{~m}^{-2} \mathrm{a}^{-1}$. On the west side of section M (i.e. from site 94 to Mirny station), it represents only summer snow ( $\mathrm{S}$ in Table $\mathrm{l}$ ).

Concentrations of $\mathrm{Cl}^{-}, \mathrm{Na}^{+}, \mathrm{Mg}^{2+}, \mathrm{Ca}^{2+}$ and $\mathrm{nssSO}_{4}{ }^{2-}$ over the ITAE route, as well as their fluxes in section $\mathrm{M}$, are shown in Figure 4. It is clear that the three ions $\mathrm{Cl}^{-}, \mathrm{Na}^{+}$, $\mathrm{Mg}^{2+}$ ) that are mainly derived from maritime aerosols display a similar distribution pattern along the ITAE route. The concentrations of the three ions are highest at sea level on the Larsen Ice Shelf, variable but quite high in the rest of sectionW (i.e. West Antarctica), lower over section M (i.e. the interior plateau of Antarctica) and even lower over section $\mathrm{S}$ (i.e. the east margin of Antarctica). Fluxes of these three ions also show a similar pattern of distribution, decreasing from west to east over section $\mathrm{M}$. Of the fluxes of the three ions, $\mathrm{Na}^{+}$and $\mathrm{Mg}^{2+}$ decrease more sharply than $\mathrm{Cl}^{-}$.

The composition and concentration of impurities in snow over Antarctica depend largely on the sources of the air masses, the transport distance and compositional changes along the transport route.

The average altitude of the initial segment of the ITAE route in West Antarctica is relatively low. This coastal region, located adjacent to the South Atlantic, is a primary source of water vapor and provides large amounts of maritime impurities to the snow of West Antarctica. The flat open area from the Ross-Bellingshausen-Weddell Sea southward to the interior continental plateau is the most favorable region in Antarctica for water-vapor transfer to inland regions. Previous aerosol studies have revealed that air masses coming

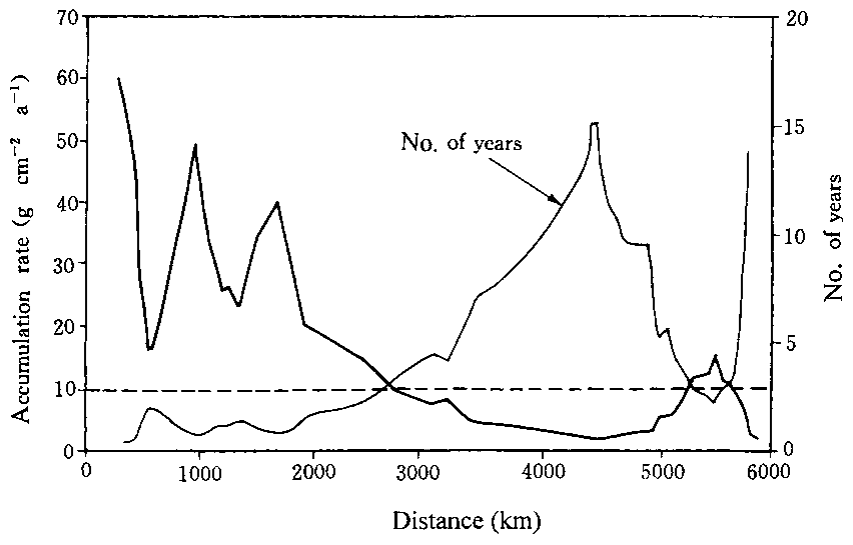

\section{Fig. 3. Annual-layer number within $1 \mathrm{~m}$ deep snow and annual mean accumulation rate along the ITAE traverse route.}

from the mid-latitudes and the sub-Antarctic Ocean are sporadically transported towards the inland plateau and even reach South Pole (Bodhaine and others, 1986). These "sodium storms" bring large quantities of sea salt to the central ice sheet.

These events are most frequent in late winter/early spring, accompanying the breakdown of the circumpolar vortex (Shaw, 1988). Hogan and others (1990) recognized that the large quantity of maritime aerosols is associated with large-
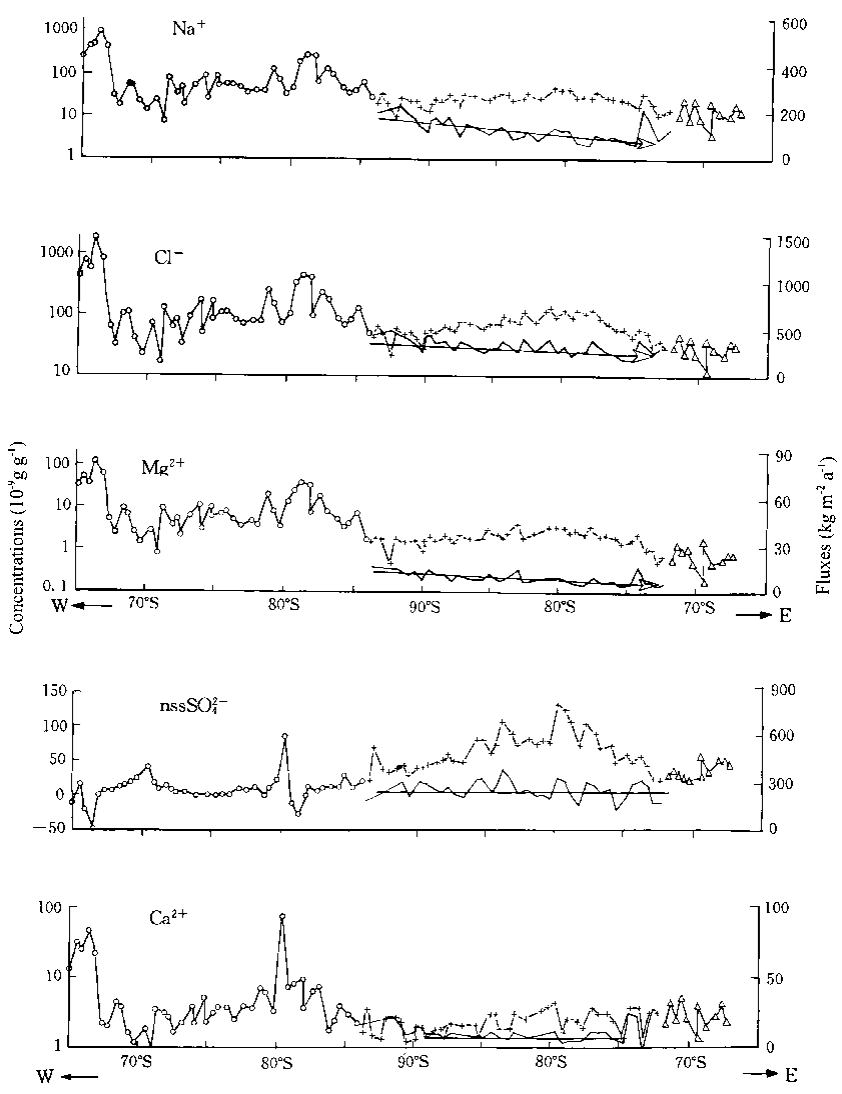

Fig. 4. Distribution of the concentrations and fluxes of $\mathrm{CT}$, $\mathrm{Na}^{+}, \mathrm{Mg}^{2+}, \mathrm{Ca}^{2+}$ and nss $\mathrm{SO}_{4}{ }^{2-}$ along the ITAE route. Circles represent the section mainly of winter snow; crosses the multi-year snow; and triangles the section mainly of summer snow. Left ordinate: the measured concentration (in exponent) of surface snow. Right ordinate: the calculated fluxes $\left(\mathrm{kg} \mathrm{m}^{-2} \mathrm{a}^{-1}\right)$ for multi-year snow only. Abscissa: the latitudes of the sampling sites. 
scale increases in temperature. Meteorological evidence shows that the precipitation season over West Antarctica is mainly during October-December (Schwerdtfeger, 1984). The route of transport for these air masses towards the South Pole coincides approximately with the route of the ITAE, and the "storm events" season occurs at the same period the ITAE team passed through West Antarctica. It is therefore not surprising that the sea-salt ions $\left(\mathrm{Cl}^{-}, \mathrm{Na}^{+}, \mathrm{Mg}^{2+}\right.$ and $\left.\mathrm{ssSO}_{4}{ }^{2-}\right)$ show high concentration in the West Antarctic segment of the route. The concentration peaks at Larsen Ice Shelf, where the elevation is close to that of sea surface.

At the other end of the ITAE route, the concentration of the sea-salt ions decreases. If the precipitation to this region is mainly derived from the adjacent ocean, i.e. the South Indian Ocean, the concentration of $\mathrm{Cl}^{-}, \mathrm{Na}^{+}$and $\mathrm{Mg}^{2+}$ should increase. Apart from a very narrow strip in coastal East Antarctica, water vapor in the regions of East Antarctica above $2000 \mathrm{~m}$ a.s.l. has clearly not come from the South Indian Ocean. It is probably derived from the vapor channel from the Ross-Bellingshausen-Weddell Sea towards the interior, especially from the Weddell Sea direction. Evidence for this inference is as follows:

(1) The central part of the East Antarctic plateau, where the altitude is over $3000 \mathrm{~m}$, is dominated by a polar cold high pressure all year round. This is an effective obstruction to air masses from the South Indian Ocean intruding up to the inland plateau.

(2) The elevation is low in the coastal regions of East Antarctica, but increases sharply inland. It rises to $>2000 \mathrm{~m}$ a.s.l. in a distance of a few tens of $\mathrm{km}$. The rapid topographic change favors the formation of orographic precipitation, and thus leads to a loss of impurities from the narrow coastal regions of East Antarctica. As noted by Delmas and Boutron (1978), deposition of coarse-sized sea-salt aerosols in Antarctic snow rapidly decreases with distance inland or elevation, and comprises only about $10 \%$ of the total sulfate deposition at inland sites in Antarctica.

(3) The fluxes of $\mathrm{Cl}^{-}, \mathrm{Na}^{+}$and $\mathrm{Mg}^{2+}$ display a decreasing trend from west to east in section $\mathrm{M}$ of the ITAE route (Fig. 3), indicating that sea-salt aerosol is mainly derived from the direction of the Weddell Sea. During transport from the coastal ocean area towards the South Pole, large particles tend to deposit in the initial segment of the route, leading to high concentrations and fluxes of sea-salt ions, such as $\mathrm{Cl}^{-}, \mathrm{Na}^{+}, \mathrm{Mg}^{2+}$ and $\mathrm{ssSO}_{4}{ }^{2-}$. But in section $\mathrm{M}$, few large particles remain in the air, and the aerosol amount largely decreases. The fluxes of the four ions decrease eastward. In fact, the interior of the Antarctic Plateau is one of the driest regions on Earth.

(4) $\mathrm{Ca}^{2+}$ does not show an eastward flux decreasing in the interior plateau. Except for a few high values, $\mathrm{Ca}^{2+}$ concentrations are very low and maintain a rather constant flux level in section M. Remote continental areas are believed to be the main sources of $\mathrm{Ca}^{2+}$ in snow of the East Antarctic plateau. The low values of $\mathrm{Ca}^{2+}$ in snow of the interior plateau represent background values for Antarctica.

High values of calcium in section W can be partly attributed to sea salt. Extremely high values are formed close to the bare rocks or mountains along the ITAE route. For instance, widespread bare bedrock and nunataks over the
Larsen Ice Shelf are responsible for the high concentrations of $\mathrm{Ca}^{2+}$ in this region. Also, calcium content at site 39 is $10-$ 20 times higher than at the adjacent sites, because site 39 is located downwind from Patriot Hills.

Sources of $\mathrm{nsSSO}_{4}{ }^{2-}$ are complicated. They may include crustal, volcanic and anthropogenic as well as marine biotic sources. In Figure 4, nssSO ${ }_{4}^{2-}$ shows high concentrations in interior Antarctica and a constant distribution of fluxes over that segment of the route. This is quite similar to the distribution of $\mathrm{Ca}^{2+}$ fluxes, which implies that their distribution in the atmosphere over the high Antarctic Plateau is uniform. Because of the high altitude of this segment of the route ( $>3600 \mathrm{~m}$ a.s.l.) we suggest that the majority of $\mathrm{Ca}^{2+}$ and nss $\mathrm{SO}_{4}{ }^{2-}$ may be derived from the upper troposphere.

\section{GONGLUDING REMARKS}

Direct observational and instrumental records in Antarctica are relatively scarce and cover a very short history. Any study of the spatial and temporal complexity of the climate system over this isolated continent is worthwhile. Up to now, the recovery of surface snow samples and ice cores has clearly been insufficient to cover the entire Antarctic continent, and this has obstructed the interpretation of even its most recent (decades to centuries) climatic and environmental history. Fortunately, the International Trans-Antarctic Scientific Expedition (ITASE) has had as a primary aim the improvement of the coverage. Future studies across the ITASE traverse net covering the whole continent will provide more detailed information on surface snow chemistry.

\section{ACKNOWLEDGEMENTS}

This work was supported by the Chinese Academy of Sciences (KZ951-A1-205) and the Ministry of Science and Technology (98-927) of China. Support was also provided by the National Committee for Antarctic Research of China, the National Committee of Science and Technology of China, and the National Natural Science Foundation of China. Partial support was provided by U.S. National Science Foundation grant DPP-8919190 and U.S. Air Force contract AFOSR 88-0065. The authors thank the members of ITAE and the staff of the offices of ITAE for their help and logistic work.

\section{REFERENGES}

Barkov, N. I. 1975. Snow accumulation along the Mirny-Vostok profile, 1970-1973. Antarct. J. U.S., 19(20), 56-57.

Bodhaine, B. A., J.J. Deluisi, J. M. Harris, P. Houmere and S. Bauman. 1986. Aerosol measurements at the South Pole. Tellus, 38B(3-4), 223-235.

Cameron, R. L., E. Picciotto, H. S. Kane and J. Gliozzi. 1968. Glaciology on the Queen Maud Land traverse, 1964-1965 South Pole-Pole of Relative Inaccessibility. Ohio State Univ. Inst. Polar Stud. Rep. 23.

Crary, A. P. 1963. Results of the United States traverses in East Antarctica, 1958-1961. IGY Glaciol. Rep. Ser. 7.

Delmas, R. and C. Boutron. 1978. Sulfate in Antarctic snow: spatio-temporal distribution. Atmos. Environ., 12(1-3), 723-728.

Drewry, D. J. 1983. The surface of the Antarctic ice sheet. In Drewry, D.J., ed. Antarctica: glaciological and geophysical folio. Cambridge, University of Cambridge. Scott Polar Research Institute, Sheet 2.

Giovinetto, M. B. and C. Bull. 1987. Summary and analyses of surface mass balance compilations for Antarctica, 1960-1985. Byrd Polar Res. Cent. Rep. 1.

Hamilton, W. L. and M. E. O'Kelley. 1971. Investigation of particulate matter in Antarctic firn. In Crary, A.P., ed. Antarctic snow and ice studies II. Washington, DC, American Geophysical Union, 355-362. (Antarctic Research Series 16.) 
Hogan, A.W., W. G. Egan and J. A. Samson. 1990. Seasonal variation of some constituents of Antarctic tropospheric air. Geophys. Res. Lett., $17(12), 2365-2368$.

Jouzel, J., L. Merlivat, J. R. Petit and C. Lorius. 1983. Climatic information over the last century deduced from a detailed isotopic record in the South Pole snow. F. Geophys. Res., 88(C4), 2693-2703.

Jouzel, J., J. R. Petit and D. Raynaud. 1990. Palaeoclimatic information from ice cores: the Vostok records. Trans. R. Soc. Edinburgh, Ser. Earth Sci., 81 (4), 349-355.

Kamiyama, K., Y. Ageta and Y. Fujii. 1989. Atmospheric and depositional environments traced from unique chemical compositions of snow over an inland high plateau, Antarctica. F. Geophys. Res., 94(D15), 18,515-18,519.

Kotlyakov, V. M., K. S. Losev and I. A. Loseva. 1978. The ice budget of Antarctica. Polar Geogr., 2 (4), 251-262.

Legrand, M. 1987. Chemistry of Antarctic snow and ice. F. Phys. (Paris), 48, Colloq. Cl, 77-86. (Supplément au 3.)

Legrand, M. and R. J. Delmas. 1985. Spatial and temporal variations of snow chemistry in Terre Adélie (East Antarctica). Ann. Glaciol., 7, 20-25.

Lipenkov, V.Ya. 1980. Nekotorye osobennosti nakopleniya snega na profile Mirnyy-Vostok [Features of snow accumulation at the Mirnyy-Vostok profile]. Inform. Byull. Sov. Antarkt. Eksped. 100, 59-60.

Lorius, C., L. Merlivat and R. Hagemann. 1970. Fluctuations of the average deuterium content of precipitation in Antarctica. Sov. Antarct. Exped. Inf. Bull., 7(6), 513-516.

Mayewski, P. A. and M. Legrand. 1990. Recent increase in nitrate concentration of Antarctic snow. Nature, 346(6281), 258-260.
Mulvaney, R. and E.W. Wolff. 1994. Spatial variability of the major chemistry of the Antarctic ice sheet. Ann. Glaciol., 20, 440-447.

Peel, D. A. and H. B. Clausen. 1982. Oxygen-isotope and total beta-radioactivity measurements on $10 \mathrm{~m}$ ice cores from the Antarctic Peninsula. 7. Glaciol., 28 (98), 43-55.

Qin Dahe. 1992. [The modern climatic and environmental record in the surface snow of the Antarctic ice sheet.] (Ph.D. thesis, Chinese Academy of Sciences. Lanzhou Institute of Glaciology and Geocryology.) [In Chinese with English abstract.]

Qin Dahe, E. J. Zeller and G. A. M. Dreschhoff. 1992. The distribution of nitrate content in the surface snow of the Antarctic ice sheet along the route of the 1990 International Trans-Antarctic Expedition. F. Geophys. Res., 97(A5), 6277-6284.

Qin Dahe, J. R. Petit, J. Jouzel and M. Stievenard. 1994. Distribution of stable isotopes in surface snow along the route of the 1990 International Trans-Antarctica Expedition. F. Glaciol., 40(134), 107-118.

Schwerdtfeger, W. 1970. The climate of the Antarctic. In Orvig, S., ed. Climates of the polar regions. New York, Elsevier, 253-355.

Schwerdtfeger, W. 1984. Weather and climate of the Antarctic. Amsterdam, Elsevier. (Developments in Atmospheric Science 15.)

Shaw, G. E. 1988. Antarctic aerosols: a review. Rev. Geophys., 26(1), 89-112.

Shimizu, H. 1964. Glaciological studies in West Antarctica, 1960-62. In Mellor, M., ed. Antarctic snow and ice studies. Washington, DC, American Geophysical Union, 37-64. (Antarctic Research Series 2.)

Taylor, L. D. 1965. Glaciological studies on the South Pole traverse, 1962 1963. Ohio State Univ. Inst. Polar Stud. Rep. 17. 